\title{
On innovations of $n$-dimensional integral-type inequality on time scales
}

\author{
Lütfi Akın ${ }^{1 *}$ (D)
}

\section{"Correspondence:}

lutfiakin@artuklu.edu.tr

1 Department of Business

Administration, Mardin Artuklu

University, 47100 Mardin, Turkey

\section{Springer}

\begin{abstract}
Integral-type inequalities and dynamic equations have an important place in time scales. In this paper, we present some innovations of n-dimensional Minkowski's integral-type inequality on time scales via $\diamond_{\alpha}$-integral.

MSC: 47B38; 34N05; 35A23

Keywords: Minkowski integral-type inequality; Time scales; Integral inequalities; Diamond $-\alpha$ integral
\end{abstract}

\section{Introduction}

For more than a quarter century, the theory of time scales, whose founder was German mathematician Stefan Hilger [1], played an important role in differential calculus, difference calculus, and quantum calculus. Later, this theory was quickly developed by many mathematicians, who added many innovations to the literature by using integral-type inequalities and dynamic equations on time scales [2-12]. Wong et al. [6, 7] expressed some integral equations on time scales. Ozkan et al. [10] demonstrated extensions of some integral inequalities on time scales. Yang [13] obtained a extension of $\diamond_{\alpha}$-integral Hölder's inequality. Georgiev et al. [14] demonstrated two dimensional integral inequalities on time scales. Anderson $[15,16]$ demonstrated some dynamic integral inequalities in two independent variables on time scales. Tuna and Kutukcu [17] reached some general conclusions about Hardy's integral inequalities by using Hölder inequalities with delta integral on time scales. Chen [18] demonstrated some generalizations of the Minkowski's integral inequality. Akın [19] showed new properties of fractional inequalities by using fractional maximal integral operators and synchronous functions on time scales. Recently, numerous applications have had an accelerating effect on the development of mathematical inequalities. These applications also attracted the attention of researchers from various disciplines, for example, quantum mechanics, phsical problems, wave equations, heat transfer, and economic problems [20-24].

The organization of this paper is as follows. In Sect. 2, we give necessary definitions, lemmas, and theorems. In Sect. 3, we demonstrate some innovations of $n$-dimensional Minkowski's integral-type inequalities on time scales via $\diamond_{\alpha}$-integral.

(c) The Author(s) 2021. This article is licensed under a Creative Commons Attribution 4.0 International License, which permits use sharing, adaptation, distribution and reproduction in any medium or format, as long as you give appropriate credit to the original author(s) and the source, provide a link to the Creative Commons licence, and indicate if changes were made. The images or other third party material in this article are included in the article's Creative Commons licence, unless indicated otherwise in a credit line to the material. If material is not included in the article's Creative Commons licence and your intended use is not permitted by statutory regulation or exceeds the permitted use, you will need to obtain permission directly from the copyright holder. To view a copy of this licence, visit http://creativecommons.org/licenses/by/4.0/. 


\section{Mathematical background}

Let us give basic information about time scales in general. For more detailed information, we refer the readers to [1-38].

The time scale $\mathbb{T}$ is a nonempty closed subset of $\mathbb{R}$. $[a, b]$ is an arbitrary interval on time scale $\mathbb{T}$, and by $[a, b]_{\mathbb{T}}$ we denote $[a, b] \cap \mathbb{T}$.

Definition 2.1 ([31]) The mappings $\sigma, \rho: \mathbb{T} \rightarrow \mathbb{T}$ defined by $\sigma(t)=\inf \{s \in \mathbb{T}: s>t\}, \rho(t)=$ $\sup \{s \in \mathbb{T}: s>t\}$ for $t \in \mathbb{T}$. Respectively, $\sigma(t)$ is the forward jump operator and $\rho(t)$ is the backward jump operator.

If $\sigma(t)>t$, then $t$ is right-scattered, and if $\sigma(t)=t$, then $t$ is called right-dense. If $\rho(t)<t$, then $t$ is left-scattered, and if $\rho(t)=t$, then $t$ is called left-dense.

Definition 2.2([31]) The mappings $\mu, \vartheta: \mathbb{T} \rightarrow \mathbb{R}^{+}$defined as $\mu(t)=\sigma(t)-t, \vartheta(t)=t-\rho(t)$ are called graininess mappings.

If $\mathbb{T}$ has a left-scattered maximum $m$, then $\mathbb{T}^{k}=\mathbb{T}-\{m\}$. Otherwise, $\mathbb{T}^{k}=\mathbb{T}$. Briefly,

$$
\mathbb{T}^{k}= \begin{cases}\mathbb{T} \backslash(\rho \sup \mathbb{T}, \sup \mathbb{T}] & \text { if } \sup \mathbb{T}<\infty \\ \mathbb{T} & \text { if } \sup \mathbb{T}=\infty\end{cases}
$$

Similarly,

$$
\mathbb{T}_{k}= \begin{cases}\mathbb{T} \backslash[\inf \mathbb{T}, \sigma(\inf \mathbb{T})], & |\inf \mathbb{T}|<\infty \\ \mathbb{T}, & \inf \mathbb{T}=-\infty\end{cases}
$$

Let $h: \mathbb{T} \rightarrow \mathbb{R}$, and let $t \in \mathbb{T}^{k}(t \neq \min \mathbb{T})$. If $h$ is $\Delta$-differentiable at point $t$, then $h$ is continuous at point $t$, and if $h$ is left continuous at point $t$ and $t$ is right-scattered, then $h$ is $\Delta$-differentiable at point $t$, and

$$
h^{\Delta}(t)=\frac{h^{\sigma}(t)-h(t)}{\mu(t)} .
$$

Let $t$ be right-dense. If $h$ is $\Delta$-differentiable at point $t$ and $\lim _{s \rightarrow t} \frac{h(t)-h(s)}{t-s}$, then

$$
h^{\Delta}(t)=\lim _{s \rightarrow t} \frac{h(t)-h(s)}{t-s},
$$

and if $h$ is $\Delta$-differentiable at point $t$, then $h^{\sigma}(t)=h(t)+\mu(t) h^{\Delta}(t)$.

Remark 2.3 If $\mathbb{T}=\mathbb{R}$, then $h^{\Delta}(t)=h^{\prime}(t)$, and if $\mathbb{T}=\mathbb{Z}$, then $h^{\Delta}(t)$ reduces to $\Delta h(t)$.

Let $n \in N$. For $i=\{1,2, \ldots, n\}$, we denote by $\mathbb{T}_{i}$ a time scale.

Definition 2.4 ([14]) The set $\aleph^{n}=\mathbb{T}_{1} \times \mathbb{T}_{2} \times \cdots \times \mathbb{T}_{n}=\left\{t=\left(t_{1}, t_{2}, \ldots, t_{n}\right): t_{i} \in \mathbb{T}_{i}, i=\right.$ $1,2, \ldots, n\}$ is called an $n$-dimensional time scale. 
Definition 2.5 ([14]) For $a=\left(a_{1}, a_{2}, \ldots, a_{n}\right) \in \mathbb{R}^{n}$ and $b=\left(b_{1}, b_{2}, \ldots, b_{n}\right) \in \mathbb{R}^{n}$, we write $a \geq b$ whenever $a_{i} \geq b_{i}$ for all $i=1,2, \ldots, n$.

Definition 2.6 ([25]) If $H: \mathbb{T} \rightarrow \mathbb{R}$ is a $\Delta$-antiderivative of $h: \mathbb{T} \rightarrow \mathbb{R}$, then $H^{\Delta}=h(t)$ for all $t \in \mathbb{T}$, and we define the $\Delta$-integral of $h$ by

$$
\int_{s}^{t} h(\tau) \Delta \tau=H(t)-H(s)
$$

for $s, t \in \mathbb{T}$.

Proposition 2.7 ([25]) Let $u, v, \alpha, t \in \mathbb{T}$ and $c \in \mathbb{R}$. If $f(\gamma)$ and $g(\gamma)$ are $\Delta$-integrable functions on $[u, v]_{\mathbb{T}}$, then the following statements are valid:

(i) $\int_{u}^{t}[f(\gamma)+g(\gamma)] \Delta \gamma=\int_{u}^{t} f(\gamma) \Delta \gamma+\int_{u}^{t} g(\gamma) \Delta \gamma$,

(ii) $\int_{u}^{t} c f(\gamma) \Delta \gamma=c \int_{u}^{t} f(\gamma) \Delta \gamma$,

(iii) $\int_{u}^{t} f(\gamma) \Delta \gamma=-\int_{t}^{u} f(\gamma) \Delta \gamma$,

(iv) $\int_{u}^{t} f(\gamma) \Delta \gamma=\int_{u}^{v} f(\gamma) \Delta \gamma+\int_{v}^{t} f(\gamma) \Delta \gamma$,

(v) $\int_{u}^{u} f(\gamma) \Delta \gamma=0$.

Lemma 2.8 ([25]) Let $u, v, \alpha, t \in \mathbb{T}$ with $u<v$. Suppose that $h(\gamma), g(\gamma)$ are $\Delta$-integrable functions on $[u, v]_{\mathbb{T}}$. Then we have

(a) If $h(\gamma) \geq 0$ for all $\gamma \in[u, v]_{\mathbb{T}}$, then $\int_{u}^{v} h(\gamma) \Delta \gamma \geq 0$,

(b) If $h(\gamma) \leq g(\gamma)$ for all $\gamma \in[u, v]_{\mathbb{T}}$, then $\int_{u}^{v} h(\gamma) \Delta \gamma \leq \int_{u}^{v} g(\gamma) \Delta \gamma$,

(c) If $h(\gamma) \geq 0$ for all $\gamma \in[u, v]_{\mathbb{T}}$, then $h(\gamma)=0$ iff $\int_{u}^{v} h(\gamma) \Delta \gamma=0$.

Definition 2.9 ([25]) Let $h: \mathbb{T}_{k} \rightarrow \mathbb{R}$ be $\nabla$-differentiable at $t \in \mathbb{T}_{k}$. If $\varepsilon>0$, then there exists a neighborhood $V$ of $t$ such that

$$
\left|h(\rho(t))-h(s)-h^{\nabla}(t)(\rho(t)-s)\right| \leq \varepsilon|\rho(t)-s|
$$

for all $s \in V$.

Definition 2.10 ([25]) Let $H: \mathbb{T} \rightarrow \mathbb{R}$ be a $\nabla$-antiderivative of $h: \mathbb{T} \rightarrow \mathbb{R}$. Then we define

$$
\int_{s}^{t} h(\tau) \nabla \tau=H(t)-H(s)
$$

for all $s, t \in \mathbb{T}$.

Let $f(t)$ be differentiable on $\mathbb{T}$ for all $t \in \mathbb{T}$. Then we define $f^{\diamond_{\alpha}}(t)$ by

$$
f^{\diamond \alpha}(t)=\alpha f^{\Delta}(t)+(1-\alpha) f^{\nabla}(t)
$$

for $0 \leq \alpha \leq 1$.

Proposition 2.11 ([25]) Iff, $h: \mathbb{T} \rightarrow \mathbb{R}$ are $\diamond_{\alpha}$-differentiable for all $\alpha, t \in \mathbb{T}$, then

(i) $f+h: \mathbb{T} \rightarrow \mathbb{R}$ is $\diamond_{\alpha}$-differentiable for $t \in \mathbb{T}$ with

$$
(f+h)^{\diamond_{\alpha}}(t)=f^{\diamond_{\alpha}}(t)+h^{\diamond_{\alpha}}(t)
$$


(ii) For $k \in \mathbb{R}, k f: \mathbb{T} \rightarrow \mathbb{R}$ is $\diamond_{\alpha}$-differentiable for all $\alpha, t \in \mathbb{T}$ with

$$
(k f)^{\diamond_{\alpha}}(t)=k f^{\diamond_{\alpha}}(t)
$$

(iii) fh: $\mathbb{T} \rightarrow \mathbb{R}$ is $\diamond_{\alpha}$-differentiable for all $\alpha, t \in \mathbb{T}$ with

$$
(f h)^{\diamond_{\alpha}}(t)=f^{\diamond_{\alpha}}(t) h(t)+\alpha f^{\sigma}(t) h^{\Delta}(t)+(1-\alpha) f^{\rho}(t) h^{\nabla}(t) .
$$

Definition 2.12 ([25]) For $\alpha, b, t \in \mathbb{T}$ and $f: \mathbb{T} \rightarrow \mathbb{R}$, we have

$$
\int_{b}^{t} f(\delta) \diamond_{\alpha} \delta=\alpha \int_{b}^{t} f(\delta) \Delta \delta+(1-\alpha) \int_{b}^{t} f(\delta) \nabla \delta
$$

for $0 \leq \alpha \leq 1$.

Definition 2.13 ([31, p. 6]) If $f \in C_{r d}(\mathbb{T}, \mathbb{R})$ and $t \in \mathbb{T}^{k}$, then we have

$$
\int_{t}^{\sigma(t)} f(\tau) \Delta \tau=\mu(t) f(t)
$$

Theorem 2.14 ([31, Theorem 1.1.2]) Iff is $\Delta$-integrable on $[a, b]$, then $|f|$ is $\Delta$-integrable on $[a, b]$, and $\left|\int_{a}^{b} f(\gamma) \Delta \gamma\right| \leq \int_{a}^{b}|f(\gamma)| \Delta \gamma$.

Theorem 2.15 ([38]) If two functions $g, h: I \rightarrow \mathbb{R}$ are $\Delta$-integrable on $I=[a, b] \in \mathbb{T}$ with $0<l \leq g^{p}, h^{p} \leq L<\infty$. If $p>1$, then we have

$$
\left(\int_{a}^{b}|g(\gamma)|^{p} \Delta \gamma\right)^{\frac{1}{p}}+\left(\int_{a}^{b}|h(\gamma)|^{p} \Delta \gamma\right)^{\frac{1}{p}} \leq 2\left(\frac{L}{l}\right)^{\frac{1}{p}}\left(\int_{a}^{b}|g(\gamma)+h(\gamma)|^{p} \Delta \gamma\right)^{\frac{1}{p}} .
$$

\section{Main results}

In this section, we state and prove our main results.

Theorem 3.1 If two mappings $g, h: I \rightarrow \mathbb{R}$ are $\diamond_{\alpha}$-integrable on $I=[a, b] \in \mathbb{T}$ with $0<l \leq$ $g^{p}, h^{p} \leq L<\infty, p>1$, then we have

$$
\begin{aligned}
& \left(\int_{a}^{b}|g(\gamma)|^{p} \diamond_{\alpha} \gamma\right)^{\frac{1}{p}}+\left(\int_{a}^{b}|h(\gamma)|^{p} \diamond_{\alpha} \gamma\right)^{\frac{1}{p}} \\
& \quad \leq 2\left(\frac{p}{(p-1)} \frac{L}{l}\right)^{\frac{1}{p}}\left(\int_{a}^{b}|g(\gamma)+h(\gamma)|^{p} \nabla_{\alpha} \gamma\right)^{\frac{1}{p}} .
\end{aligned}
$$

Proof We know that (Theorem 3.1, [38]) if $0<l \leq g^{p} \leq L<\infty$, then

$$
l^{\frac{1}{p}} \leq g \leq L^{\frac{1}{p}} .
$$

Similarly, if $0<l \leq h^{p} \leq L<\infty$, then

$$
l^{\frac{1}{p}} \leq h \leq L^{\frac{1}{p}}
$$


Multiplying both sides of inequalities (2) and (3) by $\left(\int_{a}^{b}|g(\gamma)|^{p} \diamond_{\alpha} \gamma\right)^{\frac{1}{p}}$ and $\left(\int_{a}^{b}|h(\gamma)|^{p} \diamond_{\alpha} \gamma\right)^{\frac{1}{p}}$, respectively, we have

$$
\begin{aligned}
(p-1)^{\frac{1}{p}} l^{\frac{1}{p}}\left(\int_{a}^{b}|g(\gamma)|^{p} \diamond_{\alpha} \gamma\right)^{\frac{1}{p}} & \leq L^{\frac{1}{p}}\left(\int_{a}^{b}|g(\gamma)|^{p} \diamond_{\alpha} \gamma\right)^{\frac{1}{p}} \\
& \leq p^{\frac{1}{p}} L^{\frac{1}{p}}\left(\int_{a}^{b}|g(\gamma)+h(\gamma)|^{p} \diamond_{\alpha} \gamma\right)^{\frac{1}{p}}
\end{aligned}
$$

and

$$
\begin{aligned}
(p-1)^{\frac{1}{p}} l^{\frac{1}{p}}\left(\int_{a}^{b}|h(\gamma)|^{p} \diamond_{\alpha} \gamma\right)^{\frac{1}{p}} & \leq L^{\frac{1}{p}}\left(\int_{a}^{b}|h(\gamma)|^{p} \diamond_{\alpha} \gamma\right)^{\frac{1}{p}} \\
& \leq p^{\frac{1}{p}} L^{\frac{1}{p}}\left(\int_{a}^{b}|g(\gamma)+h(\gamma)|^{p} \diamond_{\alpha} \gamma\right)^{\frac{1}{p}} .
\end{aligned}
$$

Now, if we add inequalities (4) and (5) to each other, then we have

$$
\begin{aligned}
& (p-1)^{\frac{1}{p}} l^{\frac{1}{p}}\left[\left(\int_{a}^{b}|g(\gamma)|^{p} \diamond_{\alpha} \gamma\right)^{\frac{1}{p}}+\left(\int_{a}^{b}|g(\gamma)|^{p} \diamond_{\alpha} \gamma\right)^{\frac{1}{p}}\right] \\
& \leq 2 p^{\frac{1}{p}} L^{\frac{1}{p}}\left(\int_{a}^{b}|g(\gamma)+h(\gamma)|^{p} \diamond_{\alpha} \gamma\right)^{\frac{1}{p}} .
\end{aligned}
$$

Thus we have proved inequality (1).

Theorem 3.2 Let $g, h:\left[a_{1}, b_{1}\right] \times\left[a_{1}, b_{1}\right] \times \cdots \times\left[a_{i}, b_{i}\right] \rightarrow \mathbb{R}$ be rd-continuous for $I=$ $\left[a_{i}, b_{i}\right] \in \mathbb{T}_{i}, 1 \leq i \leq n$. If $p>1$, then we have

$$
\begin{gathered}
\left(\int_{a_{1}}^{b_{1}} \int_{a_{2}}^{b_{2}} \ldots \int_{a_{i}}^{b_{i}}\left|g\left(\gamma_{1}, \gamma_{2}, \ldots, \gamma_{i}\right)+h\left(\gamma_{1}, \gamma_{2}, \ldots, \gamma_{i}\right)\right|^{p} \nabla_{\alpha} \gamma_{i}\right)^{\frac{1}{p}} \\
\leq \frac{p}{(p-1)} 2^{1-\frac{1}{p}}\left[\left(\int_{a_{1}}^{b_{1}} \int_{a_{2}}^{b_{2}} \ldots \int_{a_{i}}^{b_{i}}\left|g\left(\gamma_{1}, \gamma_{2}, \ldots, \gamma_{i}\right)\right|^{p} \nabla_{\alpha} \gamma_{i}\right)^{\frac{1}{p}}\right. \\
\left.+\left(\int_{a_{1}}^{b_{1}} \int_{a_{2}}^{b_{2}} \ldots \int_{a_{i}}^{b_{i}}\left|h\left(\gamma_{1}, \gamma_{2}, \ldots, \gamma_{i}\right)\right|^{p} \diamond_{\alpha} \gamma_{i}\right)^{\frac{1}{p}}\right]
\end{gathered}
$$

for $1 \leq i \leq n$.

Proof Let $g, h:\left[a_{1}, b_{1}\right] \times\left[a_{1}, b_{1}\right] \times \cdots \times\left[a_{i}, b_{i}\right] \rightarrow \mathbb{R}$ be $r d$-continuous and finite for $\left[a_{i}, b_{i}\right] \in \mathbb{T}_{i} \subset \mathbb{R}$. We know that [36]

$$
|g(\gamma, \theta)+h(\gamma, \theta)| \leq|g(\gamma, \theta)|+|h(\gamma, \theta)|
$$

Taking the $p$ th power of both sides, we obtain

$$
\left|g\left(\gamma_{1}, \gamma_{2}, \ldots, \gamma_{i}\right)+h\left(\gamma_{1}, \gamma_{2}, \ldots, \gamma_{i}\right)\right|^{p} \leq 2^{p-1}\left[\left|g\left(\gamma_{1}, \gamma_{2}, \ldots, \gamma_{i}\right)\right|^{p}+\left|h\left(\gamma_{1}, \gamma_{2}, \ldots, \gamma_{i}\right)\right|^{p}\right]
$$

for $1 \leq i \leq n$. 
Taking the two-dimensional $\diamond_{\alpha}$-integral over $\left[a_{i}, b_{i}\right]$ of both sides of inequality (9) and the power of order $\frac{1}{p}$, we obtain

$$
\begin{gathered}
\left(\int_{a_{1}}^{b_{1}} \int_{a_{2}}^{b_{2}} \ldots \int_{a_{i}}^{b_{i}}\left|g\left(\gamma_{1}, \gamma_{2}, \ldots, \gamma_{i}\right)+h\left(\gamma_{1}, \gamma_{2}, \ldots, \gamma_{i}\right)\right|^{p} \nabla_{\alpha} \gamma_{i}\right)^{\frac{1}{p}} \\
\leq \frac{p}{(p-1)} 2^{1-\frac{1}{p}}\left[\left(\int_{a_{1}}^{b_{1}} \int_{a_{2}}^{b_{2}} \ldots \int_{a_{i}}^{b_{i}}\left|g\left(\gamma_{1}, \gamma_{2}, \ldots, \gamma_{i}\right)\right|^{p} \diamond_{\alpha} \gamma_{i}\right)^{\frac{1}{p}}\right. \\
\left.\quad+\left(\int_{a_{1}}^{b_{1}} \int_{a_{2}}^{b_{2}} \ldots \int_{a_{i}}^{b_{i}}\left|h\left(\gamma_{1}, \gamma_{2}, \ldots, \gamma_{i}\right)\right|^{p} \diamond_{\alpha} \gamma_{i}\right)^{\frac{1}{p}}\right] .
\end{gathered}
$$

Thus the proof of Theorem 3.2 is completed.

Theorem 3.3 Let $M\left(\left(\gamma_{1}, \gamma_{2}, \ldots, \gamma_{i}\right),\left(\theta_{1}, \theta_{2}, \ldots, \theta_{i}\right)\right), g\left(\gamma_{1}, \gamma_{2}, \ldots, \gamma_{i}\right), h\left(\theta_{1}, \theta_{2}, \ldots, \theta_{i}\right), \Phi\left(\gamma_{1}, \gamma_{2}\right.$, $\left.\ldots, \gamma_{i}\right), \Psi\left(\theta_{1}, \theta_{2}, \ldots, \theta_{i}\right)$ be nonnegative functions, and let $g, h:\left[a_{1}, b_{1}\right] \times\left[a_{1}, b_{1}\right] \times \cdots \times$ $\left[a_{i}, b_{i}\right] \rightarrow \mathbb{R}$ be rd-continuous and finite for $\left[a_{i}, b_{i}\right] \in \mathbb{T}_{i} \subset \mathbb{R}$. Let

$$
\begin{aligned}
& H\left(\theta_{1}, \theta_{2}, \ldots, \theta_{i}\right)=\int_{a_{1}}^{b_{1}} \int_{a_{2}}^{b_{2}} \ldots \int_{a_{i}}^{b_{i}} \frac{M\left(\left(\gamma_{1}, \gamma_{2}, \ldots, \gamma_{i}\right),\left(\theta_{1}, \theta_{2}, \ldots, \theta_{i}\right)\right)}{\Psi\left(\theta_{1}, \theta_{2}, \ldots, \theta_{i}\right)^{p}} \nabla_{\alpha} \gamma_{i}, \\
& K\left(\gamma_{1}, \gamma_{2}, \ldots, \gamma_{i}\right)=\int_{a_{1}}^{b_{1}} \int_{a_{2}}^{b_{2}} \ldots \int_{a_{i}}^{b_{i}} \frac{M\left(\left(\gamma_{1}, \gamma_{2}, \ldots, \gamma_{i}\right),\left(\theta_{1}, \theta_{2}, \ldots, \theta_{i}\right)\right)}{\Phi\left(\gamma_{1}, \gamma_{2}, \ldots, \gamma_{i}\right)^{p}} \diamond_{\alpha} \theta_{i}
\end{aligned}
$$

for $\left[a_{i}, b_{i}\right] \in \mathbb{T}_{i} \subset \mathbb{R}$ and $1 \leq i \leq n$. If $p>1$, then we have the following inequalities:

$$
\begin{aligned}
& \left(\int_{a_{1}}^{b_{1}} \int_{a_{2}}^{b_{2}} \ldots \int_{a_{i}}^{b_{i}} M\left(\left(\gamma_{1}, \gamma_{2}, \ldots, \gamma_{i}\right),\left(\theta_{1}, \theta_{2}, \ldots, \theta_{i}\right)\right)\right. \\
& \times\left|g\left(\gamma_{1}, \gamma_{2}, \ldots, \gamma_{i}\right)+h\left(\theta_{1}, \theta_{2}, \ldots, \theta_{i}\right)\right|^{p} \diamond_{\alpha} \gamma_{i} \\
& +\int_{a_{1}}^{b_{1}} \int_{a_{2}}^{b_{2}} \ldots \int_{a_{i}}^{b_{i}} M\left(\left(\gamma_{1}, \gamma_{2}, \ldots, \gamma_{i}\right),\left(\theta_{1}, \theta_{2}, \ldots, \theta_{i}\right)\right) \\
& \left.\times\left|g\left(\gamma_{1}, \gamma_{2}, \ldots, \gamma_{i}\right)+h\left(\theta_{1}, \theta_{2}, \ldots, \theta_{i}\right)\right|^{p} \diamond_{\alpha} \theta_{i}\right)^{\frac{1}{p}} \\
& \leq \frac{p}{(p-1)} 2^{1-\frac{1}{p}}\left[\left(\int_{a_{1}}^{b_{1}} \int_{a_{2}}^{b_{2}} \ldots \int_{a_{i}}^{b_{i}} \Phi\left(\gamma_{1}, \gamma_{2}, \ldots, \gamma_{i}\right)^{p} K\left(\gamma_{1}, \gamma_{2}, \ldots, \gamma_{i}\right)\right.\right. \\
& \left.\times\left|g\left(\gamma_{1}, \gamma_{2}, \ldots, \gamma_{i}\right)\right|^{p} \diamond_{\alpha} \gamma_{i}\right)^{\frac{1}{p}} \\
& +\left(\int_{a_{1}}^{b_{1}} \int_{a_{2}}^{b_{2}} \ldots \int_{a_{i}}^{b_{i}} \Psi\left(\theta_{1}, \theta_{2}, \ldots, \theta_{i}\right)^{p} H\left(\theta_{1}, \theta_{2}, \ldots, \theta_{i}\right)\right. \\
& \left.\left.\times\left|h\left(\theta_{1}, \theta_{2}, \ldots, \theta_{i}\right)\right|^{p} \diamond_{\alpha} \theta_{i}\right)^{\frac{1}{p}}\right] \\
& \int_{a_{1}}^{b_{1}} \int_{a_{2}}^{b_{2}} \ldots \int_{a_{i}}^{b_{i}} H\left(\theta_{1}, \theta_{2}, \ldots, \theta_{i}\right)^{1-p} \Psi\left(\theta_{1}, \theta_{2}, \ldots, \theta_{i}\right)^{p(1-p)} \\
& \times\left(\int_{a_{1}}^{b_{1}} \int_{a_{2}}^{b_{2}} \ldots \int_{a_{i}}^{b_{i}} M\left(\left(\gamma_{1}, \gamma_{2}, \ldots, \gamma_{i}\right),\left(\theta_{1}, \theta_{2}, \ldots, \theta_{i}\right)\right) g\left(\gamma_{1}, \gamma_{2}, \ldots, \gamma_{i}\right) \diamond_{\alpha} \gamma_{i}\right)^{p} \nabla_{\alpha} \theta_{i}
\end{aligned}
$$




$$
\begin{aligned}
\leq & \frac{p}{(p-1)} \int_{a_{1}}^{b_{1}} \int_{a_{2}}^{b_{2}} \ldots \int_{a_{i}}^{b_{i}} \Phi\left(\gamma_{1}, \gamma_{2}, \ldots, \gamma_{i}\right)^{p} K\left(\gamma_{1}, \gamma_{2}, \ldots, \gamma_{i}\right) \\
& \times\left|g\left(\gamma_{1}, \gamma_{2}, \ldots, \gamma_{i}\right)\right|^{p} \diamond_{\alpha} \gamma_{i} \\
\int_{a_{1}}^{b_{1}} \int_{a_{2}}^{b_{2}} \ldots \int_{a_{i}}^{b_{i}} K\left(\gamma_{1}, \gamma_{2}, \ldots, \gamma_{i}\right)^{1-p} \Phi\left(\gamma_{1}, \gamma_{2}, \ldots, \gamma_{i}\right)^{p(1-p)} & \\
& \times\left(\int_{a_{1}}^{b_{1}} \int_{a_{2}}^{b_{2}} \ldots \int_{a_{i}}^{b_{i}} M\left(\left(\gamma_{1}, \gamma_{2}, \ldots, \gamma_{i}\right),\left(\theta_{1}, \theta_{2}, \ldots, \theta_{i}\right)\right)\right. \\
& \left.\times\left|g\left(\theta_{1}, \theta_{2}, \ldots, \theta_{i}\right)\right| \nabla_{\alpha} \theta_{i}\right)^{p} \nabla_{\alpha} \gamma_{i} \\
\leq & \frac{p}{(p-1)} \int_{a_{1}}^{b_{1}} \int_{a_{2}}^{b_{2}} \ldots \int_{a_{i}}^{b_{i}} \Psi\left(\theta_{1}, \theta_{2}, \ldots, \theta_{i}\right)^{p} H\left(\theta_{1}, \theta_{2}, \ldots, \theta_{i}\right) \\
& \times\left|g\left(\theta_{1}, \theta_{2}, \ldots, \theta_{i}\right)\right|^{p} \diamond_{\alpha} \theta_{i} .
\end{aligned}
$$

Proof First, let us prove inequality (10).

Let $g, h:\left[a_{1}, b_{1}\right] \times\left[a_{1}, b_{1}\right] \times \cdots \times\left[a_{i}, b_{i}\right] \rightarrow \mathbb{R}$ be $r d$-continuous and finite for $\left[a_{i}, b_{i}\right] \in$ $\mathbb{T}_{i} \subset \mathbb{R}$, and let $M\left(\left(\gamma_{1}, \gamma_{2}, \ldots, \gamma_{i}\right),\left(\theta_{1}, \theta_{2}, \ldots, \theta_{i}\right)\right)$ be nonnegative kernel functions. Let us consider the equation

$$
\begin{aligned}
\left(\int_{a_{1}}^{b_{1}} \int_{a_{2}}^{b_{2}} \ldots \int_{a_{i}}^{b_{i}} M\left(\left(\gamma_{1}, \gamma_{2}, \ldots, \gamma_{i}\right),\left(\theta_{1}, \theta_{2}, \ldots, \theta_{i}\right)\right)\right. \\
\quad \times\left|g\left(\gamma_{1}, \gamma_{2}, \ldots, \gamma_{i}\right)+h\left(\theta_{1}, \theta_{2}, \ldots, \theta_{i}\right)\right|^{p} \diamond_{\alpha} \gamma_{i} \\
\quad+\int_{a_{1}}^{b_{1}} \int_{a_{2}}^{b_{2}} \ldots \int_{a_{i}}^{b_{i}} M\left(\left(\gamma_{1}, \gamma_{2}, \ldots, \gamma_{i}\right),\left(\theta_{1}, \theta_{2}, \ldots, \theta_{i}\right)\right) \\
\left.\quad \times\left|g\left(\gamma_{1}, \gamma_{2}, \ldots, \gamma_{i}\right)+h\left(\theta_{1}, \theta_{2}, \ldots, \theta_{i}\right)\right|^{p} \diamond_{\alpha} \theta_{i}\right)^{\frac{1}{p}} \\
\quad \frac{p}{(p-1)}\left(\left(\int_{a_{1}}^{b_{1}} \int_{a_{2}}^{b_{2}} \ldots \int_{a_{i}}^{b_{i}} M\left(\left(\gamma_{1}, \gamma_{2}, \ldots, \gamma_{i}\right),\left(\theta_{1}, \theta_{2}, \ldots, \theta_{i}\right)\right)\right.\right. \\
\left.\quad \times\left|\frac{g\left(\gamma_{1}, \gamma_{2}, \ldots, \gamma_{i}\right) \Phi\left(\gamma_{1}, \gamma_{2}, \ldots, \gamma_{i}\right)}{\Phi\left(\gamma_{1}, \gamma_{2}, \ldots, \gamma_{i}\right)}+\frac{h\left(\gamma_{1}, \gamma_{2}, \ldots, \gamma_{i}\right) \Psi\left(\theta_{1}, \theta_{2}, \ldots, \theta_{i}\right)}{\Psi\left(\theta_{1}, \theta_{2}, \ldots, \theta_{i}\right)}\right|^{p} \diamond_{\alpha} \gamma_{i}\right) \\
\quad+\left(\int_{a_{1}}^{b_{1}} \int_{a_{2}}^{b_{2}} \ldots \int_{a_{i}}^{b_{i}} M\left(\left(\gamma_{1}, \gamma_{2}, \ldots, \gamma_{i}\right),\left(\theta_{1}, \theta_{2}, \ldots, \theta_{i}\right)\right)\right. \\
\left.\left.\quad \times\left|\frac{g\left(\gamma_{1}, \gamma_{2}, \ldots, \gamma_{i}\right) \Phi\left(\gamma_{1}, \gamma_{2}, \ldots, \gamma_{i}\right)}{\Phi\left(\gamma_{1}, \gamma_{2}, \ldots, \gamma_{i}\right)}+\frac{h\left(\gamma_{1}, \gamma_{2}, \ldots, \gamma_{i}\right) \Psi\left(\theta_{1}, \theta_{2}, \ldots, \theta_{i}\right)}{\Psi\left(\theta_{1}, \theta_{2}, \ldots, \theta_{i}\right)}\right|^{p} \vartheta_{\alpha} \theta_{i}\right)\right)^{\frac{1}{p}} .
\end{aligned}
$$

Applying the Minkowski inequality to the right side of inequality (13), by Theorem 3.2 we complete the proof of inequality (10).

Now let us prove inequality (11). Let us consider the equation

$$
\begin{aligned}
& \int_{a_{1}}^{b_{1}} \int_{a_{2}}^{b_{2}} \ldots \int_{a_{i}}^{b_{i}} H\left(\theta_{1}, \theta_{2}, \ldots, \theta_{i}\right)^{1-p} \Psi\left(\theta_{1}, \theta_{2}, \ldots, \theta_{i}\right)^{p(1-p)} \\
& \quad \times\left(\int_{a_{i}}^{b_{i}} M\left(\left(\gamma_{1}, \gamma_{2}, \ldots, \gamma_{i}\right),\left(\theta_{1}, \theta_{2}, \ldots, \theta_{i}\right)\right)\left|g\left(\gamma_{1}, \gamma_{2}, \ldots, \gamma_{i}\right)\right| \nabla_{\alpha} \gamma_{i}\right)^{p} \nabla_{\alpha} \theta_{i}
\end{aligned}
$$




$$
\begin{aligned}
= & \frac{p}{(p-1)} \int_{a_{1}}^{b_{1}} \int_{a_{2}}^{b_{2}} \ldots \int_{a}^{b} M\left(\left(\gamma_{1}, \gamma_{2}, \ldots, \gamma_{i}\right),\left(\theta_{1}, \theta_{2}, \ldots, \theta_{i}\right)\right)^{1-p} \\
& \times\left(\int_{a_{1}}^{b_{1}} \int_{a_{2}}^{b_{2}} \ldots \int_{a_{i}}^{b_{i}} M\left(\left(\gamma_{1}, \gamma_{2}, \ldots, \gamma_{i}\right), \theta\right)\left|g\left(\gamma_{1}, \gamma_{2}, \ldots, \gamma_{i}\right)\right| \nabla_{\alpha} \gamma_{i}\right)^{p} \nabla_{\alpha} \theta_{i} .
\end{aligned}
$$

Applying the Hölder inequality to the right side of equality (14), we obtain

$$
\begin{aligned}
\int_{a_{1}}^{b_{1}} \int_{a_{2}}^{b_{2}} \ldots \int_{a_{i}}^{b_{i}} H\left(\theta_{1}, \theta_{2}, \ldots, \theta_{i}\right)^{1-p} \Psi\left(\theta_{1}, \theta_{2}, \ldots, \theta_{i}\right)^{p(1-p)} \\
\quad \times\left(\int_{a_{1}}^{b_{1}} \int_{a_{2}}^{b_{2}} \ldots \int_{a_{i}}^{b_{i}} M\left(\left(\gamma_{1}, \gamma_{2}, \ldots, \gamma_{i}\right),\left(\theta_{1}, \theta_{2}, \ldots, \theta_{i}\right)\right)\left|g\left(\gamma_{1}, \gamma_{2}, \ldots, \gamma_{i}\right)\right| \nabla_{\alpha} \gamma_{i}\right)^{p} \nabla_{\alpha} \theta_{i} \\
\leq \frac{p}{(p-1)} \int_{a_{1}}^{b_{1}} \int_{a_{2}}^{b_{2}} \ldots \int_{a_{i}}^{b_{i}} M\left(\left(\gamma_{1}, \gamma_{2}, \ldots, \gamma_{i}\right),\left(\theta_{1}, \theta_{2}, \ldots, \theta_{i}\right)\right) \\
\quad \times\left[\left(\int_{a_{1}}^{b_{1}} \int_{a_{2}}^{b_{2}} \ldots \int_{a_{i}}^{b_{i}}\left|g\left(\gamma_{1}, \gamma_{2}, \ldots, \gamma_{i}\right)\right|^{p} \diamond_{\alpha} \gamma_{i}\right)^{\frac{1}{p}}\left(\int_{a_{1}}^{b_{1}} \int_{a_{2}}^{b_{2}} \ldots \int_{a_{i}}^{b_{i}} \nabla_{\alpha} \gamma_{i}\right)^{\frac{1}{q}}\right] \nabla_{\alpha} \theta_{i} \\
\leq \frac{p}{(p-1)} \int_{a_{1}}^{b_{1}} \int_{a_{2}}^{b_{2}} \ldots \int_{a_{i}}^{b_{i}} M\left(\left(\gamma_{1}, \gamma_{2}, \ldots, \gamma_{i}\right),\left(\theta_{1}, \theta_{2}, \ldots, \theta_{i}\right)\right) \\
\quad \times\left[\left(\int_{a_{1}}^{b_{1}} \int_{a_{2}}^{b_{2}} \ldots \int_{a_{i}}^{b_{i}}\left|g\left(\gamma_{1}, \gamma_{2}, \ldots, \gamma_{i}\right)\right|^{p} \diamond_{\alpha} \gamma_{i}\right)^{\frac{1}{p}}\right] \nabla_{\alpha} \theta_{i} \\
=\frac{p}{(p-1)} \int_{a_{1}}^{b_{1}} \int_{a_{2}}^{b_{2}} \ldots \int_{a_{i}}^{b_{i}} \Phi\left(\gamma_{1}, \gamma_{2}, \ldots, \gamma_{i}\right)^{p} K\left(\gamma_{1}, \gamma_{2}, \ldots, \gamma_{i}\right)\left|g\left(\gamma_{1}, \gamma_{2}, \ldots, \gamma_{i}\right)\right|^{p} \diamond_{\alpha} \gamma_{i} .
\end{aligned}
$$

Thus we have completed the proof of Theorem 3.3. The reader can see the proof of inequality (12), similar to the proof of inequality (11).

Remark 3.4 Let $f\left(\theta_{1}, \theta_{2}, \ldots, \theta_{i}\right), g\left(\gamma_{1}, \gamma_{2}, \ldots, \gamma_{i}\right), h\left(\theta_{1}, \theta_{2}, \ldots, \theta_{i}\right), \Phi\left(\gamma_{1}, \gamma_{2}, \ldots, \gamma_{i}\right), \Psi\left(\theta_{1}, \theta_{2}\right.$, $\ldots, \theta_{i}$ ) be nonnegative functions. If we put

$$
M\left(\left(\gamma_{1}, \gamma_{2}, \ldots, \gamma_{i}\right),\left(\theta_{1}, \theta_{2}, \ldots, \theta_{i}\right)\right)= \begin{cases}f\left(\theta_{1}, \theta_{2}, \ldots, \theta_{i}\right), & \left(\gamma_{1}, \gamma_{2}, \ldots, \gamma_{i}\right) \leq\left(\theta_{1}, \theta_{2}, \ldots, \theta_{i}\right), \\ 0, & \left(\gamma_{1}, \gamma_{2}, \ldots, \gamma_{i}\right)>\left(\theta_{1}, \theta_{2}, \ldots, \theta_{i}\right),\end{cases}
$$

and

$$
M\left(\left(\gamma_{1}, \gamma_{2}, \ldots, \gamma_{i}\right),\left(\theta_{1}, \theta_{2}, \ldots, \theta_{i}\right)\right)= \begin{cases}f\left(\theta_{1}, \theta_{2}, \ldots, \theta_{i}\right), & \left(\gamma_{1}, \gamma_{2}, \ldots, \gamma_{i}\right)>\left(\theta_{1}, \theta_{2}, \ldots, \theta_{i}\right) \\ 0, & \left(\gamma_{1}, \gamma_{2}, \ldots, \gamma_{i}\right) \leq\left(\theta_{1}, \theta_{2}, \ldots, \theta_{i}\right)\end{cases}
$$

then the inequalities in Theorem 3.3 are provided.

\section{Conclusion}

Recently, the concept of inequalities in time scales has gained an important place in the scientific literature. Mathematicians have emphasized many aspects of integral inequalities. For example, transformations, inverse conversions, extensions, and so on. However, we found that little work has been done on multidimensional inequalities in time scales. In this paper, we proved some innovations of $n$-dimensional Minkowski's $\diamond_{\alpha}$-integral inequality in time scales. We think that our method is applicable to different integral-type 
inequalities as well. As a result, we predict that new versions of known inequalities may be obtained.

\author{
Acknowledgements \\ The author is grateful to the editors and reviewers for spending their precious time for the paper.
}

Funding

Not applicable.

Availability of data and materials

Not applicable.

Competing interests

The author declares that they have no competing interests.

\title{
Authors' contributions
}

The author read and approved the final manuscript.

\section{Publisher's Note}

Springer Nature remains neutral with regard to jurisdictional claims in published maps and institutional affiliations.

Received: 15 October 2020 Accepted: 13 January 2021 Published online: 03 March 2021

\section{References}

1. Hilger, S.: Ein Maßkettenkalkül mit Anwendung auf Zentrmsmannigfaltingkeiten. Ph.D. Thesis, Univarsi.Würzburg (1988)

2. Agarwal, R.P., Bohner, M., Peterson, A.: Inequalities on time scales: a survey. Math. Inequal. Appl. 4, 535-557 (2001)

3. Akin-Bohner, E., Bohner, M., Akin, F.: Pachpatte inequalities on time scales. JIPAM. J. Inequal. Pure Appl. Math. 6(1), $1-23(2005)$

4. Li, W.N.: Nonlinear integral inequalities in two independent variables on time scales. Adv. Differ. Equ. 2011, Article ID 283926 (2011)

5. Anastassiou, G.A.: Principles of delta fractional calculus on time scales and inequalities. Math. Comput. Model. 52(3-4), 556-566 (2010)

6. Wong, F.H., Yeh, C.C., Yu, S.L., Hong, C.H.: Young's inequality and related results on time scales. Appl. Math. Lett. 18 983-988 (2005)

7. Wong, F.H., Yeh, C.C., Lian, W.C.: An extension of Jensen's inequality on time scales. Adv. Dyn. Syst. Appl. 1(1), 113-120 (2006)

8. Kuang, J.: Applied Inequalities. Shandong Science Press, Jinan (2003)

9. Ucar, D., Hatipoglu, V.F., Akincali, A.: Fractional integral inequalities on time scales. Open J. Math. Sci. 2(1), 361-370 (2018)

10. Ozkan, U.M., Sarikaya, M.Z., Yildirim, H.: Extensions of certain integral inequalities on time scales. Appl. Math. Lett. 21 993-1000 (2008)

11. Tian, J.F., Ha, M.H.: Extensions of Hölder-type inequalities on time scales and their applications. J. Nonlinear Sci. Appl. 10, 937-953 (2017)

12. Kac, V., Cheung, P.: Quantum Calculus. Springer, New York (2002)

13. Yang, W.G.: A functional generalization of diamond- $\alpha$ integral Hölder's inequality on time scales. Appl. Math. Lett. 23, 1208-1212 (2010)

14. Georgiev, S.G., Khadekar, G., Kumar, P.: Two dimensional integral inequalities on time scales (2020). arXiv:2003.05309. arXiv preprint, arxiv.org

15. Anderson, D.R.: Dynamic double integral inequalities in two independent variables on time scales. J. Math. Inequal. 2(2), 163-184 (2008)

16. Anderson, D.R.: Nonlinear dynamic integral inequalities in two independent variables on time scale pairs. Adv. Dyn. Syst. Appl. 3(1), 113 (2008)

17. Tuna, A., Kutukcu, S.: Some integral inequalities on time scales. Appl. Math. Mech. 29(1), 23-28 (2008)

18. Chen, G.-S.: Some improvements of Minkowski's integral inequality on time scales. J. Inequal. Appl. 2013, 318 (2013) $1-6$

19. Akın, L:: On the fractional maximal delta integral type inequalities on time scales. Fractal Fract. 4(2), 1-10 (2020)

20. Spedding, V.: Taming nature's numbers. New Sci. 179(2404), 28-31 (2003)

21. Tisdell, C.C., Zaidi, A.: Basic qualitative and quantitative results for solutions to nonlinear dynamic equations on time scales with an application to economic modelling. Nonlinear Anal. 68(11), 3504-3524 (2008)

22. Bohner, M., Heim, J., Liu, A.: Qualitative analysis of Solow model on time scales. J. Concr. Appl. Math. 13(3-4), 183-197 (2015)

23. Brigo, D., Mercurio, F.: Discrete time vs continuous time stock-price dynamics and implications for option pricing. Finance Stoch. 4, 147-159 (2000)

24. Seadawy, A.R., Iqbal, M., Lu, D.: Nonlinear wave solutions of the Kudryashov-Sinelshchikov dynamical equation in mixtures liquid-gas bubbles under the consideration of heat transfer and viscosity. J. Taibah Univ. Sci. 13(1), 1060-1072 (2019)

25. Bohner, M., Peterson, A.: Dynamic Equations on Time Scales, an Introduction with Applications. Birkhäuser, Boston (2001) 
26. Sheng, Q., Fadag, M., Henderson, J., Davis, J.M.: An exploration of combined dynamic derivatives on time scales and their applications. Nonlinear Anal., Real World Appl. 7(3), 395-413 (2006)

27. Qi, F.: Several integral inequalities. RGMIA Res. Rep. Collect. 2(7) Art. 9, 1039-1042 (1999)

28. Qi, F.: Several integral inequalities. J. Inequal. Pure Appl. Math. 1(2), 2-7 (2000)

29. Hilger, S.: Analysis on measure chains - a unified approach to continuous and discrete calculus. Results Math. 18 , 18-56 (1990)

30. Agarwal, R.P., O'Regan, D., Saker, S.H.: Dynamic Inequalities on Time Scales. Springer, Heidelberg (2014)

31. Agarwal, R.P., O’Regan, D., Saker, S.H.: Hardy Type Inequalities on Time Scales. Springer, Cham (2016)

32. Bohner, M., Guseinov, G.S.: Multiple Lebesgue integration on time scales. Adv. Differ. Equ. 2006, Article ID 026391 (2006)

33. Chen, G., Wei, C.: A functional generalization of diamond- $\alpha$ integral Dresher's inequality on time scales. Adv. Differ Equ. 2014, Article ID 324 (2014)

34. Yin, L., Qi, F.: Some integral inequalities on time scales. Results Math. 64(3), 371-381 (2013)

35. Qi, F., Li, A.-J., Zhao, W.-Z., Niu, D.-W., Cao, J.: Extensions of several integral inequalities. J. Inequal. Pure Appl. Math. 7(3), $1-6(2006)$

36. Balcı, M.: Genel Matematik. BalcıYayınları, Ankara (2008). ISBN 978-975-6683-00-2

37. Akın, L.: On some results of weighted Hölder type inequality on time scales. Middle East J. Sci. 6(1), 15-22 (2020)

38. Akın, L.: On some integral type inequality on time scales. In: 3rd International e-Conference on Mathematical Advances and Applications, Yildiz Technical University, Istanbul, Turkey, June 24-27, 2020 (accepted)

\section{Submit your manuscript to a SpringerOpen ${ }^{0}$ journal and benefit from:}

- Convenient online submission

Rigorous peer review

Open access: articles freely available online

High visibility within the field

Retaining the copyright to your article

Submit your next manuscript at $\gg$ springeropen.com 